Supporting information

\title{
Synergism of Multi-Component Catalysis: One-dimensional Pt-Rh-Pd Nanochain Catalysts for Efficient Methanol Oxidation
}

Yang Jiang\#, Yuanyuan Guo*, Yanyan Zhou, Shuolei Deng, Li Hou*, Yunfeng Niu, Tifeng Jiao*

Hebei Key Laboratory of Applied Chemistry, Hebei Key Laboratory of Heavy Metal

Deep-Remediation in Water and Resource Reuse, School of Environmental and Chemical Engineering, Yanshan University, Qinhuangdao 066004, P. R. China

${ }^{*}$ Corresponding authors.

E-mail address: holy@ysu.edu.cn (L. Hou); tfjiao@ysu.edu.cn (T. Jiao);

\#These authors contributed equally to this work. 


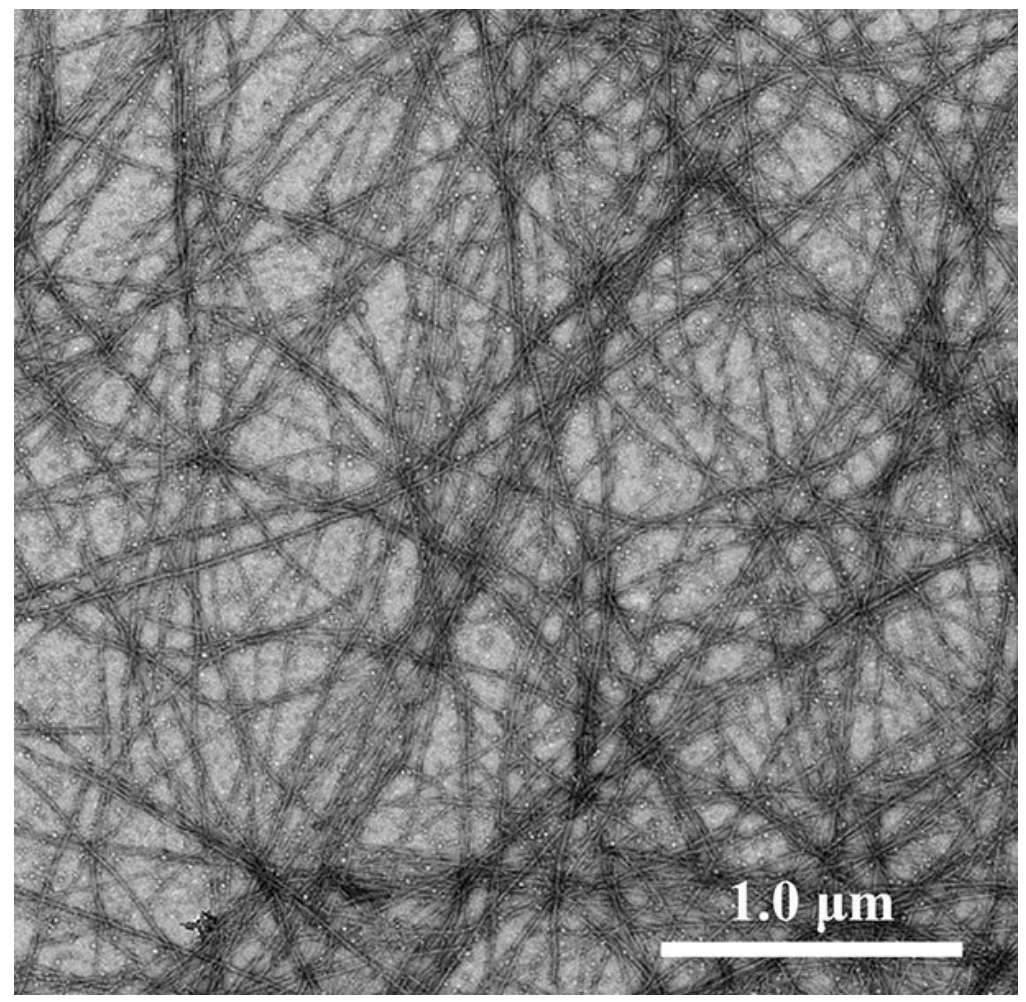

Figure S1. TEM image of negatively stained insulin fibers.
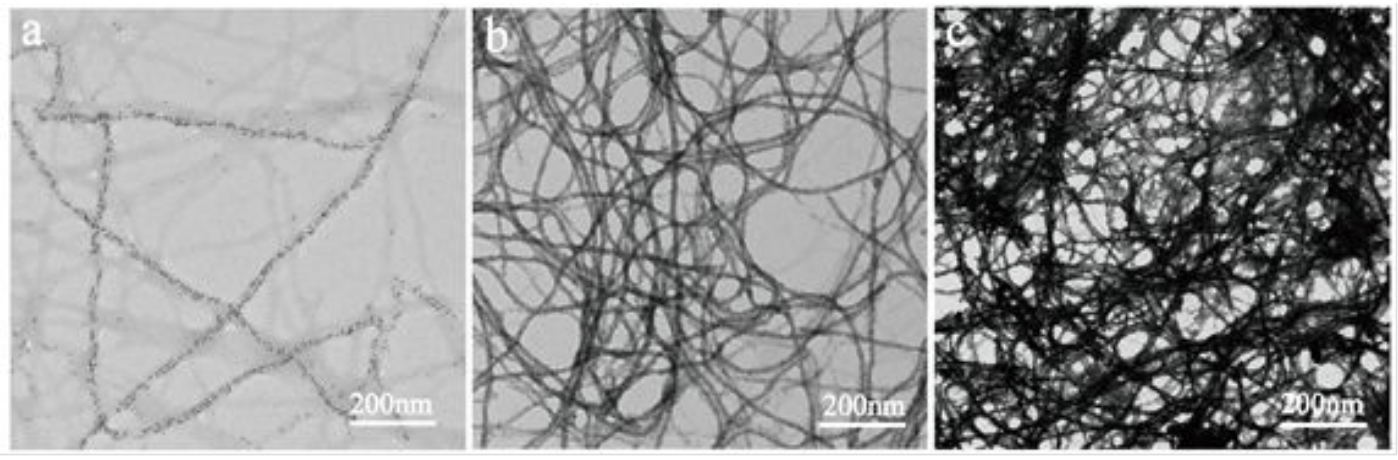

Figure S2. TEM images of Pt-Rh-Pd NCs with different mixed salt additions: a, 30 $\mu \mathrm{L} ; \mathrm{b}, 60 \mu \mathrm{L} ; \mathrm{c}, 90 \mu \mathrm{L}$. 

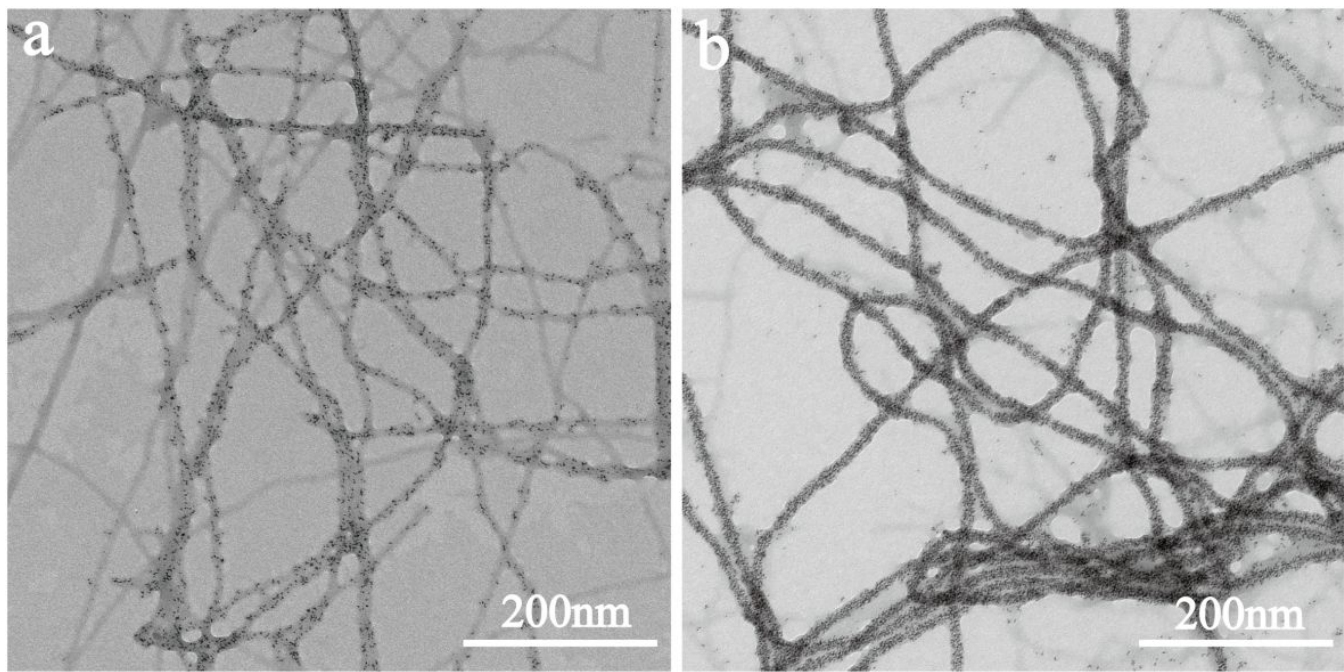

Figure S3. TEM images of Pt-Rh-Pd NCs with different reducing agents: a, DMAB; b, $\mathrm{NaBH}_{4}$.

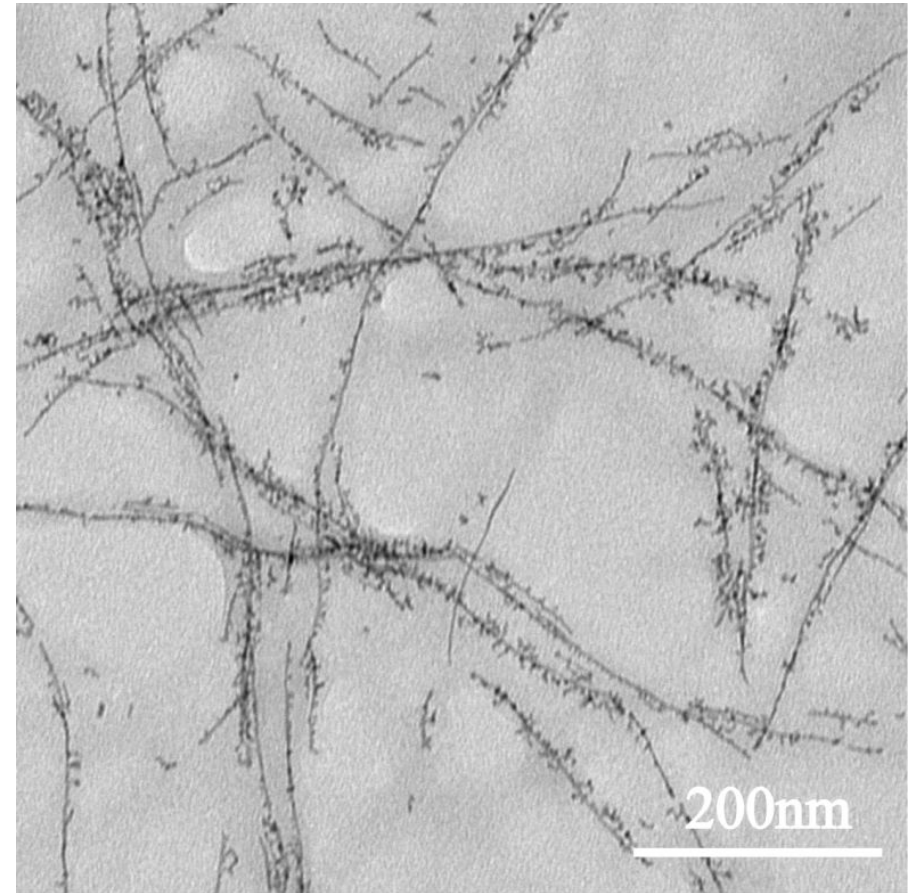

Figure S4. TEM image of Pt-Rh NCs 
Table S1. Values of $E_{f}, J_{f}, E_{b}, J_{b}$ and $J_{f} / J_{b}$ of different Pt-based nanomaterials in the electrocatalytic oxidation of methanol.

\begin{tabular}{cccc} 
& Pt-Rh-Pd NCs & Pt-Rh NCs & Commercial Pt/C \\
\hline $\mathbf{E}_{\mathbf{f}} / \mathbf{V}$ & 0.52 & 0.58 & 0.63 \\
$\mathbf{J}_{\mathbf{f}} / \mathbf{m A ~ \mathbf { ~ m } ^ { - 2 }}$ & 1.48 & 1.07 & 0.89 \\
$\mathbf{E}_{\mathbf{b}} / \mathbf{V}$ & 0.37 & 0.39 & 0.40 \\
$\mathbf{J}_{\mathbf{b}} / \mathbf{m A ~ c m} \mathbf{~ c m}^{-2}$ & 0.26 & 0.45 & 0.78 \\
$\mathbf{J}_{\mathbf{f}} / \mathbf{J}_{\mathbf{b}}$ & 5.69 & 2.38 & 1.14 \\
\hline
\end{tabular}

